\title{
Banco de sementes no solo em sistemas de cultivo lavoura-pastagem
}

\author{
Fernanda Satie Ikeda ${ }^{(1)}$, Danielle Mitja ${ }^{(2)}$, Lourival Vilela(1) e Ricardo Carmona $^{(3)}$
}

\begin{abstract}
(1)Embrapa Cerrados, BR 020, Km 18, Caixa Postal 08223, CEP 73310-970 Planaltina, DF. E-mail: satieikeda@zipmail.com.br, Ivilela@cpac.embrapa.br (2)IRD/Embrapa Cerrados, US Espace 140 et UMR 137, Domaine de Lavalette, Centre IRD de Montpellier, 911, Avenue Agropolis, BP 64501 - F-34394, Montpellier Cedex 5, França. E-mail: danielle.mitja@ird.fr (3)Universidade de Brasília, Fac. de Agronomia e Medicina Veterinária, Caixa Postal 04508, CEP 70910-970 Brasília, DF. E-mail: rcarmona@unb.br
\end{abstract}

\begin{abstract}
Resumo - O objetivo deste trabalho foi avaliar o efeito de sistemas de cultivo, do preparo do solo e dos níveis de adubação sobre o banco de sementes, em solos de áreas submetidas a: três sistemas de cultivo - lavoura contínua (L), lavoura-pastagem-lavoura (LPL) e pastagem-lavoura-pastagem (PLP); dois sistemas de preparo do solo - convencional (C) e semeadura direta (D); dois níveis de adubação - manutenção (1) e corretiva gradual (2); e uma área de pastagem contínua, com preparo convencional e adubação corretiva gradual. No sistema de cultivo lavoura-pastagem, a densidade de sementes, excluindo-se as áreas de cultivo de LPLC1 e de LPLC2, foi menor do que nas lavouras contínuas e maior do que na pastagem contínua. Nas áreas de lavoura, o banco de sementes foi menor em áreas com semeadura direta do que com preparo convencional do solo, mas nas áreas de PLP, com adubação corretiva gradual, não houve diferença entre os sistemas de preparo do solo. A adubação causou redução na densidade de sementes apenas nas áreas de cultivo de LPLC e PLPC. A adoção de sistemas de cultivo lavoura-pastagem com semeadura direta pode auxiliar no manejo de plantas daninhas em áreas de lavoura de grãos.
\end{abstract}

Termos para indexação: adubação, Cerrado, integração lavoura-pecuária, planta daninha, preparo convencional do solo, semeadura direta.

\section{Soil seedbank in integrated crop-pasture systems}

\begin{abstract}
The objective of this study was to evaluate the effects of cultivation and tillage systems, and fertilization level, on soil seedbanks of areas submitted to: three cultivation systems - continuous crop (L), crop-pasturecrop (LPL), pasture-crop-pasture (PLP); two tillage systems - tillage (C) and no-tillage (D); two fertilization levels - maintenance (1) and gradual corrective (2); and a continuous pasture area under tillage and gradual corrective fertilization. Seed density of crop-pasture cultivation systems, was lower than in continuous crop, and higher compared to continuous pasture system, except for LPLC1 and LPLC2 cultivated areas. The seedbanks in continuous cropping areas was lower in no-tillage than in tillage system areas, but in PLP areas with gradual corrective fertilization, there was no difference between tillage systems. The fertilization level caused a reduction in seed density only in LPLC and PLPC cultivated areas. Integrated crop-pasture system with no-tillage adoption can be useful in weed management of grains crop areas.
\end{abstract}

Index terms: fertilization, Cerrado, integrated crop-livestock, weed, tillage, no-tillage.

\section{Introdução}

A integração dos sistemas de produção de grãos e pecuária vem ganhando força como alternativa para a solução de alguns problemas das lavouras e pastagens contínuas. Com essa integração, podem-se reduzir os riscos de degradação e melhorar as propriedades químicas, físicas e biológicas do solo e o potencial produtivo de grãos e forragem (Vilela et al., 2003). São possíveis diversas combinações de culturas anuais e pastagens, além de variações no preparo do solo e no manejo da fertilidade.

O manejo efetuado nos cultivos, normalmente, afeta a dinâmica de plantas daninhas no solo em áreas de lavoura contínua. No entanto, praticamente não há estudos relacionados ao efeito dos sistemas de cultivo lavoura-pastagem na dinâmica de plantas daninhas. O banco de sementes e as plantas que não são eliminadas com as práticas de manejo constituem as principais fontes de infestações futuras de plantas 
daninhas nas culturas (Ekeleme et al., 2003). Assim, no caso das lavouras contínuas, a rotação de culturas de lavoura e o preparo do solo podem modificar a composição e a densidade dos bancos de sementes (Légère et al., 2005; Sosnoskie et al., 2006). Acredita-se que o uso integrado da semeadura direta com a rotação de culturas poderia resultar na redução das infestações de plantas daninhas, ao longo dos anos, em áreas de lavoura contínua (Pereira \& Velini, 2003).

Além disso, o fornecimento adequado de nutrientes para as culturas, geralmente, aumenta a capacidade de competição das culturas com as plantas daninhas (Zimdahl, 1993). Entretanto, nem sempre há modificações significativas na densidade de sementes (Moonen \& Bàrberi, 2004), apesar de a adubação poder alterar a composição de espécies, pois algumas podem se adaptar a solos com maior fertilidade, e outras, a solos com fertilidade abaixo da necessária para a cultura (Zimdahl, 1993).

O objetivo deste trabalho foi avaliar o efeito de sistemas de cultivo, do preparo do solo e dos níveis de adubação sobre o banco de sementes no solo de áreas submetidas a quatro sistemas de cultivo.

\section{Material e Métodos}

Este estudo foi realizado em um experimento de cultivo lavoura-pastagem, instalado em 1991, na Embrapa Cerrados, Planaltina, DF. A área localiza-se em Latossolo Vermelho, textura argilosa, a 15³9'84"S e $47^{\circ} 44^{\prime} 41^{\prime \prime} \mathrm{W}$, em altitude aproximada de $1.217 \mathrm{~m}$. O clima foi classificado como Aw (Köppen), com duas estações bem definidas (seca e chuvosa), e ocorrência de períodos de estiagem durante a estação chuvosa (veranicos). A área experimental foi planejada e conduzida com o objetivo de avaliar combinações de culturas e pastagens, com acompanhamento das tendências no uso de forrageiras e de manejo cultural, no sistema de cultivo lavoura-pastagem.

Foram selecionadas 12 áreas desse experimento, submetidas a: três sistemas de cultivo - lavoura contínua (L), lavoura-pastagem-lavoura (LPL) e pastagemlavoura-pastagem (PLP); dois sistemas de preparo do solo - convencional (C) e semeadura direta (D); dois níveis de adubação - manutenção (1) e corretiva gradual (2); e uma área de pastagem contínua (P), com preparo convencional e adubação corretiva gradual. A dimensão de cada área foi de 40×100 m.

O histórico das áreas com a seqüência de culturas e o total de calcário e de fertilizantes aplicados nos dois níveis de adubação de cada sistema de cultivo encontram-se descritos na Tabela 1.

Nas áreas de lavoura, o preparo convencional do solo foi efetuado com uma gradagem pesada, após a colheita, e uma gradagem pesada seguida por uma gradagem de nivelamento, antes da semeadura. A semeadura direta foi estabelecida a partir da safra de 1995/1996, com dessecação prévia das plantas daninhas. Na safra de 2003/2004, foi semeada Brachiaria brizantha como planta de cobertura, apenas nas áreas de cultivo de LPLD, com o objetivo de formar cobertura morta para a semeadura direta no ano seguinte, de acordo com o sistema Santa Fé (Cobucci \& Portela, 2003).

Os tratos culturais foram aplicados conforme recomendações para cada cultura. Nos anos de cultivo da soja, foram utilizados os herbicidas trifluralin e imazaquin, em preparo convencional para aplicação em pré-emergência, e o glyphosate, para dessecação em semeadura direta. Em ambos os sistemas de preparo de solo, e conforme a necessidade foram utilizados os

Tabela 1. Histórico de culturas e total de calcário e fertilizantes aplicados nos sistemas de cultivo, em experimento de cultivo lavoura-pastagem.

\begin{tabular}{|c|c|c|c|c|c|c|c|c|c|c|c|c|c|}
\hline \multirow{2}{*}{$\begin{array}{l}\text { Sistema } \\
\text { de cultivo }^{(1)}\end{array}$} & \multicolumn{3}{|c|}{ Ciclos de cultura } & \multicolumn{6}{|c|}{ Adubação de manutenção } & \multicolumn{4}{|c|}{ Adubação corretiva gradual } \\
\hline & $\begin{array}{c}1991 / 1992- \\
1994 / 1995 \\
\end{array}$ & $\begin{array}{c}1995 / 1996- \\
1998 / 1999 \\
\end{array}$ & $\begin{array}{l}1999 / 2000- \\
2003 / 2004\end{array}$ & $\begin{array}{c}\text { Calcário } \\
\left(\mathrm{t} \mathrm{ha}^{-1}\right)\end{array}$ & $\mathrm{N}$ & $\begin{array}{r}\mathrm{P}_{2} \mathrm{O}_{5} \\
-\left(\mathrm{kg} \mathrm{ha}^{-1}\right) \\
\end{array}$ & $\mathrm{K}_{2} \mathrm{O}$ & $\begin{array}{l}\text { Gesso } \\
\left(\mathrm{t} \mathrm{ha}^{-1}\right) \\
\end{array}$ & $\begin{array}{l}\text { Calcário } \\
\left(\mathrm{t} \mathrm{ha}^{-1}\right)\end{array}$ & $\begin{array}{l}\mathrm{N} \\
--\cdot- \\
\end{array}$ & $\begin{array}{r}\mathrm{P}_{2} \mathrm{O}_{5} \\
\left(\mathrm{~kg} \mathrm{ha}^{-1}\right) \\
\end{array}$ & $\mathrm{K}_{2} \mathrm{O}$ & $\begin{array}{r}\text { Gesso } \\
\left(\mathrm{t} \mathrm{ha}^{-1}\right) \\
\end{array}$ \\
\hline$\overline{\mathrm{L}}$ & $\mathrm{S} / \mathrm{S} / \mathrm{M} / \mathrm{S}$ & $\mathrm{M} / \mathrm{S} / \mathrm{M} / \mathrm{S}$ & $\mathrm{S} / \mathrm{Mi} / \mathrm{S} / \mathrm{Mi} / \mathrm{S}$ & 4,4 & 120 & 594 & 466 & 0,0 & 7,5 & 235 & 1.123 & 1.037 & 2,8 \\
\hline LPL & $\mathrm{S} / \mathrm{S} / \mathrm{M} / \mathrm{S}$ & $\mathrm{A} / \mathrm{A} / \mathrm{A} / \mathrm{A}$ & $\mathrm{S} / \mathrm{Mi} / \mathrm{S} / \mathrm{Mi} / \mathrm{S}+\mathrm{B}^{*}$ & 4,4 & 40 & 406 & 318 & 0,0 & 7,5 & 85 & 768 & 723 & 2,8 \\
\hline PLP & $\mathrm{A} / \mathrm{A} / \mathrm{A} / \mathrm{A}$ & $\mathrm{M} / \mathrm{S} / \mathrm{M} / \mathrm{S}$ & $\mathrm{P} / \mathrm{P} / \mathrm{P} / \mathrm{P} / \mathrm{P}$ & 4,4 & 120 & 327 & 207 & 0,0 & 7,5 & 190 & 511 & 380 & 0,0 \\
\hline$\underline{\mathrm{P}}$ & $\mathrm{A} / \mathrm{A} / \mathrm{A} / \mathrm{A}$ & $\mathrm{A} / \mathrm{A} / \mathrm{A} / \mathrm{A}$ & $\mathrm{A} / \mathrm{Bd} / \mathrm{Bd} / \mathrm{Bd} / \mathrm{Bd}$ & - & - & - & - & - & 6,8 & 80 & 254 & 204 & 0,0 \\
\hline
\end{tabular}

${ }^{(1)}$ L: lavoura contínua; LPL: lavoura-pastagem-lavoura; PLP: pastagem-lavoura-pastagem; P: pastagem contínua. A: capim-andropógon (Andropogon gayanus); B*: braquiarão (Brachiaria brizantha), apenas na semeadura direta; Bd: capim-braquiária (B. decumbens); M: milho (Zea mays); Mi: milheto (Pennisetum americanum); P: capim-tanzânia (Panicum maximum cultivar Tanzânia); S: soja (Glycine max). 
herbicidas: haloxyfop-methyl, fluazifop-p-butyl + fomesafen, fluazifop-p-butyl, metolachlor, diclosulam ou chlorimuron-ethyl.

Nas áreas de cultivo de LPL, foram aplicadas doses reduzidas de haloxyfop-methyl, para controlar o crescimento de $B$. brizantha, de forma a reduzir a competição com a soja. Nos anos de cultivo do milho, foram utilizados os herbicidas atrazine + metolachlor. Não foram aplicados herbicidas no cultivo do milheto. As doses utilizadas seguiram as recomendações do produto. Nas pastagens contínuas, o preparo convencional foi efetuado apenas na implantação (1991) e na renovação (2000) das pastagens. Nas áreas de cultivo de PLP, o preparo ocorreu na fase de lavoura e antes do estabelecimento das pastagens. Nas pastagens contínuas, as adubações foram feitas de acordo com as recomendações para as espécies, com base nos resultados de análise do solo e de produtividade esperada. No sistema de cultivo lavoura-pastagem, a adubação residual das lavouras permitiu o estabelecimento das pastagens. Não foram utilizados herbicidas nas pastagens.

A amostragem do solo, para avaliação do banco de sementes, foi efetuada na última safra do ciclo de 1999/ 2000-2003/2004, entre meados de outubro e de novembro de 2004, depois da colheita da soja e antes do preparo para a safra seguinte.

Adotou-se uma amostragem sistemática por conglomerados, mediante a coleta de oito amostras, compostas por quatro subamostras. As coletas foram realizadas em duas linhas transversais às de cultivo, distantes $50 \mathrm{~m}$ entre si, nas profundidades de $0-5 \mathrm{~cm}$ e de 5-20 cm, que totalizaram 104 amostras para cada profundidade. A distância entre os pontos de amostragem foi de $10 \mathrm{~m}$, tendo-se desconsiderado $5 \mathrm{~m}$ de bordadura. Cada subamostra foi coletada com o auxílio de uma furadeira industrial, com serra-copos de $7,3 \mathrm{~cm}$ de diâmetro e uma haste com apoio que se acoplava aos serra-copos, para formar um trado nas áreas com solo compactado.

Depois da secagem ao ar, as amostras de solo foram homogeneizadas e distribuídas aleatoriamente, dentro de casa telada, em bandejas de plástico perfuradas, com

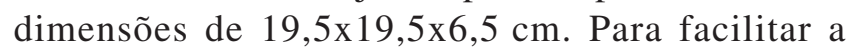
drenagem e evitar o escoamento de amostras, cada bandeja recebeu 0,3 $\mathrm{L}$ de areia esterilizada, antes de as amostras de solo serem acondicionadas. Foram distribuídas, também, oito bandejas de controle, que continham apenas areia esterilizada, para o monitoramento da contaminação externa com sementes.

Para estimular a germinação e a quebra de dormência, houve presença de luz, alternância de temperaturas e irrigações diárias na casa telada. Os indivíduos presentes em cada bandeja foram identificados com uma numeração e um morfotipo. Após seis meses de emergência e avaliação, o solo foi mantido sem irrigação, por cinco dias, e revolvido para estimular novos fluxos de emergência. A emergência de plântulas cessou ao final de aproximadamente oito meses de avaliação, que totalizou 16 levantamentos com intervalos de aproximadamente 15 dias. O número de sementes foi obtido pela soma das plântulas emergidas em cada amostra, durante todo o período.

Foram coletadas amostras de plântulas, plantas adultas e plantas com flores e/ou frutos das espécies, para confirmar a identificação botânica em herbário. Esse procedimento foi efetuado na Embrapa Recursos Genéticos e Biotecnologia e na Reserva Ecológica do IBGE. As amostras com flores e/ou frutos foram depositadas no Herbário do IBGE.

As variáveis avaliadas em cada área foram: número de espécies e densidade de sementes, nas profundidades de $0-20 \mathrm{~cm}$, e porcentagem de sementes de cada espécie, em relação à densidade encontrada em cada área. As sementes que germinaram e morreram antes de serem identificadas constaram apenas da avaliação de densidade de sementes. Os dados foram expressos em sementes por metro quadrado. Efetuou-se a análise estatística apenas com os dados de densidade de sementes, por comparação de médias pelo teste não paramétrico de Wilcoxon $(\mathrm{p}=0,05)$, no programa SAS 8.0. Não foi efetuada a análise estatística do número de espécies, pois foi considerado o somatório de todas as espécies encontradas no conjunto de amostras de cada área.

\section{Resultados e Discussão}

Não ocorreu contaminação externa de sementes, de acordo com os resultados das bandejas controle. Nos resultados das amostras, observou-se que a densidade de sementes foi menor nos sistemas de cultivo lavoura-pastagem do que nas lavouras contínuas, excluindo-se as áreas de cultivo LPLC (Tabela 2). Além disso, os sistemas de cultivo lavoura-pastagem apresentaram maior densidade de sementes do que a área de pastagem contínua. 
O menor banco de sementes na área de cultivo de LPLD (entre as áreas de lavoura com semeadura direta) e na área de pastagem contínua (entre as áreas de pastagem) deve-se, provavelmente, à presença de $B$. brizantha e de $B$. decumbens, respectivamente (Tabela 2). A influência dessas forrageiras também foi observada com a maior redução na infestação por plantas daninhas, no consórcio de $B$. brizantha com milho, e com a maior redução na infestação dessa cultura com o consórcio de $P$. maximum do que com o de B. decumbens (Severino et al., 2006).

Nas áreas de cultivo de LPLC, ocorreu maior número de culturas (5), o que levou aparentemente a um maior número de espécies de plantas daninhas, independentemente do nível de adubação, do que nas áreas de lavoura contínua ( 3 culturas) com preparo convencional (Tabela 2). Esses resultados corroboram os de outros trabalhos, em que o aumento no número de espécies de plantas daninhas também esteve associado ao aumento do número de culturas de lavouras em rotação (Bellinder et al., 2004; Sosnoskie et al., 2006). Isso está, normalmente, relacionado à aplicação de herbicidas de seletividade diferente (Sosnoskie et al., 2006). Nas áreas de cultivo de LPLC, mesmo com quatro anos de pastagens no histórico, não se observou a redução no número de espécies de plantas daninhas, verificada nas áreas de lavoura com a presença de gramínea forrageira (LPLD), em comparação às

Tabela 2. Número de sementes por metro quadrado e de espécies no solo, em 13 áreas de 40x100 m de um experimento de cultivo lavoura-pastagem, na profundidade de $0-20 \mathrm{~cm}^{(1)}$.

\begin{tabular}{lccccc}
\hline Sistema & \multicolumn{2}{c}{ Número de sementes } & & \multicolumn{2}{c}{ Número de espécies } \\
\cline { 2 - 3 } \cline { 5 - 6 } & $\begin{array}{c}\text { Adubação de } \\
\text { manutenção }\end{array}$ & $\begin{array}{c}\text { Adubação } \\
\text { corretiva gradual }\end{array}$ & & $\begin{array}{c}\text { Adubação de } \\
\text { manutenção }\end{array}$ & $\begin{array}{c}\text { Adubação } \\
\text { corretiva gradual }\end{array}$ \\
\hline LC & $23.654 \mathrm{bA}$ & $21.839 \mathrm{aA}$ & & 24 & 26 \\
LD & $10.468 \mathrm{cA}$ & $9.744 \mathrm{bA}$ & & 28 & 34 \\
LPLC & $45.747 \mathrm{aA}$ & $24.400 \mathrm{aB}$ & & 27 & 33 \\
LPLD & $2.815 \mathrm{deA}$ & $2.882 \mathrm{dA}$ & & 22 & 30 \\
PLPC & $2.389 \mathrm{eB}$ & $5.115 \mathrm{cA}$ & & 21 & 28 \\
PLPD & $5.167 \mathrm{dA}$ & $4.472 \mathrm{cA}$ & & 17 & 17 \\
PC & $-2)$ & $1.322 \mathrm{e}$ & & - & 20 \\
\hline
\end{tabular}

(1)L: lavoura contínua; LPL: lavoura-pastagem-lavoura; PL: pastagemlavoura-pastagem; P: pastagem contínua; C: preparo convencional do solo; D: semeadura direta; em cada área, foram coletadas oito amostras compostas por quatro subamostras de $7,3 \mathrm{~cm}$ de diâmetro; médias seguidas por letras iguais, minúsculas na coluna e maiúsculas na linha, não diferem entre si pelo teste de Wilcoxon, a 5\% de probabilidade; para cada comparação, utilizou-se uma DMS diferente. ${ }^{(2)}$ Área não avaliada. áreas de lavoura contínua com o mesmo preparo do solo e nível de adubação.

Nas áreas de cultivo de LPLD, a presença de B. brizantha, em apenas um ano, proporcionou o menor número de espécies, em relação às áreas de lavoura contínua com semeadura direta. Apesar de ocorrer maior número de culturas em rotação nas áreas de cultivo de PLP (4 culturas) do que nas de lavoura contínua (3 culturas) de mesmo preparo do solo e nível de adubação, ocorreu menor número de espécies de plantas daninhas nas áreas de cultivo de PLP, à exceção do cultivo de PLPC2, que apresentou maior número de espécies que LC2. Isso ocorreu, provavelmente, em razão da influência de forrageiras nessas áreas, pois normalmente ocorre menor número de espécies de plantas daninhas nas áreas de pastagens do que nas de lavoura (Gasparino et al., 2006). No caso da pastagem contínua, a rotação entre duas gramíneas forrageiras possibilitou a ocorrência de 20 espécies de plantas daninhas.

Entre as cinco espécies que mais contribuíram para a densidade total, em cada área (Tabela 3), verificou-se que Eleusine indica esteve entre aquelas que apresentaram maior porcentagem de densidade de sementes, em quase todas as áreas avaliadas. Isso ocorreu, possivelmente, em razão de sua grande capacidade de reprodução e por ser pouco exigente em relação ao solo (Kissmann, 1997). Eragrostis rufescens, Sabicea cf. brasiliensis e Schwenckia americana ocorreram com maior densidade relativa, principalmente em áreas com a presença de forrageiras. Essas espécies são nativas do bioma Cerrado (Mendonça et al., 1998) e não há relato sobre as condições ambientais preferenciais para seu desenvolvimento.

As áreas de lavoura sob semeadura direta apresentaram menos sementes no solo do que as áreas com preparo convencional (Tabela 2). O efeito da perturbação do solo sobre o tamanho do banco de sementes ainda não está bem esclarecido. Sosnoskie et al. (2006) verificaram maior densidade de sementes no solo com a semeadura direta do que no preparo convencional do solo, em áreas de lavoura contínua. Entretanto, Murphy et al. (2006) observaram que a densidade de sementes diminuiu na semeadura direta e no cultivo mínimo, mas permaneceu constante no preparo convencional, após seis anos de estudo. As menores densidades de sementes com a semeadura direta, em relação ao preparo convencional ou cultivo mínimo, têm 
sido atribuídas à elevada porcentagem de deterioração, herbivoria ou incidência de patógenos (Chauhan et al., 2006; Murphy et al., 2006). Nas áreas de cultivo de PLP com a adubação corretiva gradual, a densidade de sementes na semeadura direta não diferiu daquela encontrada no preparo convencional do solo. A falta de revolvimento do solo, durante os cinco anos da fase de pastagem no cultivo de PLP, provavelmente provocou homogeneização das áreas e, conseqüentemente, ausência de efeito do tipo de preparo no final do ciclo de cinco anos. Entretanto, nas áreas de cultivo de PLP com a adubação de manutenção, a densidade de sementes foi menor com o preparo convencional do que com a semeadura direta.

O número de espécies de plantas daninhas, nas áreas de lavoura contínua, foi maior com a semeadura direta, enquanto nas áreas de cultivo lavoura-pastagem o número de espécies foi maior com o preparo convencional do solo (Tabela 2). Além disso, as áreas de lavoura contínua com semeadura direta apresentaram o maior número de espécies, entre todas as áreas de mesmo nível de adubação. Em outros trabalhos, a redução no revolvimento do solo também esteve associada ao maior número de espécies, em áreas de lavoura contínua (Favreto \& Medeiros, 2006; Sosnoskie et al., 2006).
No caso das áreas com cultivo lavoura-pastagem, a presença de forrageiras parece ter sido, também, a causa na diferença desses resultados, normalmente observada com o preparo do solo em áreas de lavoura contínua, embora não haja na literatura referências sobre o assunto. Blainvillea rhomboidea e Desmodium sp. ocorreram com maior porcentagem de densidade em áreas com semeadura direta do que em áreas com preparo convencional (Tabela 3 ). Centratherum cf. camporum, Commelina benghalensis, Cenchrus echinatus, Digitaria ciliaris e Digitaria cf. horizontalis ocorreram em maior porcentagem de densidade em áreas com preparo convencional do que em áreas com semeadura direta. A menor densidade de sementes de C. benghalensis, em áreas com semeadura direta, devese provavelmente ao sombreamento. Além disso, D. ciliaris tem preferência por solos bem trabalhados, e D. horizontalis costuma ser uma das primeiras infestantes a aparecer após o preparo do solo (Kissmann, 1997). De acordo com Pereira \& Velini (2003), a ocorrência de C. echinatus foi reduzida ao longo de quatro anos com a semeadura direta, o que pode caracterizar uma condição desfavorável ao desenvolvimento dessa espécie. Não são encontradas informações na literatura sobre as demais espécies.

Tabela 3. Principais espécies encontradas no banco de sementes de 13 áreas de 40x100 m, de um experimento de cultivo lavoura-pastagem, na profundidade de $0-20 \mathrm{~cm}^{(1)}$.

\begin{tabular}{|c|c|c|c|c|c|c|c|c|c|c|c|c|c|}
\hline Espécie & LC1 & $\mathrm{LC} 2$ & LD1 & LD2 & LPLC1 & LPLC2 & LPLD1 & LPLD2 & PLPC1 & PLPC2 & PLPD1 & PLPD2 & $\mathrm{PC} 2$ \\
\hline & & & & & & & $-(\%)$ & & - & & & & \\
\hline Ageratum conyzoides & $39,2^{(2)}$ & $39,9^{(2)}$ & $18,5^{(2)}$ & $31,0^{(2)}$ & $44,1^{(2)}$ & $59,3^{(2)}$ & $3,4^{(2)}$ & $13,7^{(2)}$ & $4,7^{(2)}$ & $36,9^{(2)}$ & 0,9 & 2,5 & $1,7^{(2)}$ \\
\hline Cenchrus echinatus & $4,7^{(2)}$ & 2,4 & 0,4 & 0,8 & 0,1 & 0,2 & 0,2 & - & - & - & - & - & 0,5 \\
\hline Centratherum cf. camporum & $8,0^{(2)}$ & $6,3^{(2)}$ & 1,1 & 1,1 & 0,0 & - & 1,1 & 1,6 & - & - & - & - & - \\
\hline Commelina benghalensis & 0,1 & $6,8^{(2)}$ & 0,1 & 3,1 & - & 1,5 & - & 0,2 & - & 0,9 & - & 0,5 & - \\
\hline Digitaria ciliaris & 1,5 & 3,4 & 0,4 & 1,9 & $3,2^{(2)}$ & $1,9^{(2)}$ & $2,7^{(2)}$ & 0,8 & 3,1 & 0,3 & 0,1 & 0,2 & - \\
\hline Digitaria $\mathrm{cf}$. horizontalis & $14,0^{(2)}$ & $7,6^{(2)}$ & 2,2 & 1,1 & $26,9^{(2)}$ & $4,6^{(2)}$ & 0,5 & 1,0 & 0,6 & 0,1 & - & - & 0,5 \\
\hline Pennisetum spp..$^{(3)}$ & 2,3 & 2,8 & $19,4^{(2)}$ & 2,1 & 0,6 & 0,2 & 0,8 & - & - & 0,1 & - & - & - \\
\hline Blainvillea rhomboidea & 0,4 & 1,3 & $4,3^{(2)}$ & $4,3^{(2)}$ & 0,1 & 0,2 & - & 1,6 & 0,3 & 0,4 & - & - & - \\
\hline Desmodium sp. & 0,5 & 1,0 & $3,5^{(2)}$ & $10,4^{(2)}$ & - & - & - & - & - & - & - & - & - \\
\hline Nicandra physaloides & 0,1 & 1,5 & 0,2 & 2,0 & 0,1 & $3,9^{(2)}$ & - & $11,7^{(2)}$ & 1,3 & 0,3 & 0,4 & 2,7 & $1,7^{(2)}$ \\
\hline Solanum americanum & 0,1 & 3,9 & 0,1 & $3,4^{(2)}$ & 0,1 & $2,7^{(2)}$ & 1,8 & $4,6^{(2)}$ & 0,3 & $16,6^{(2)}$ & 1,0 & $9,3^{(2)}$ & $2,8^{(2)}$ \\
\hline Acanthospermum australe & 1,9 & 0,4 & 1,2 & 0,1 & $3,1^{(2)}$ & 0,2 & 2,4 & 0,2 & $12,6^{(2)}$ & $2,8^{(2)}$ & $26,4^{(2)}$ & 1,3 & 0,5 \\
\hline Eragrostis rufescens & 0,6 & 0,3 & 0,7 & - & 1,8 & 0,1 & $5,8^{(2)}$ & 0,2 & $10,0^{(2)}$ & 0,6 & $17,5^{(2)}$ & $2,8^{(2)}$ & $2,8^{(2)}$ \\
\hline Schwenckia americana & 0,3 & 0,1 & 1,1 & 2,6 & 0,1 & 0,3 & $7,4^{(2)}$ & $2,6^{(2)}$ & $12,2^{(2)}$ & $2,9^{(2)}$ & $30,2^{(2)}$ & $31,1^{(2)}$ & $1,7^{(2)}$ \\
\hline Sabicea cf. brasiliensis & - & 0,1 & 0,1 & 1,1 & 0,1 & 0,1 & - & $6,0^{(2)}$ & 2,2 & 0,4 & $5,6^{(2)}$ & $9,0^{(2)}$ & $19,7^{(2)}$ \\
\hline Eleusine indica & $6,8^{(2)}$ & $10,4^{(2)}$ & $6,1^{(2)}$ & $4,8^{(2)}$ & $8,2^{(2)}$ & $16,3^{(2)}$ & $42,5^{(2)}$ & $27,2^{(2)}$ & $18,2^{(2)}$ & $20,4^{(2)}$ & $1,9^{(2)}$ & $2,8^{(2)}$ & $2,8^{(2)}$ \\
\hline Richardia brasiliensis & $9,7^{(2)}$ & $4,5^{(2)}$ & $24,3^{(2)}$ & $12,4^{(2)}$ & $4,5^{(2)}$ & 3,3 & $6,1^{(2)}$ & 0,5 & $3,8^{(2)}$ & $2,8^{(2)}$ & $3,9^{(2)}$ & $22,7^{(2)}$ & $1,7^{(2)}$ \\
\hline Outras & 1,4 & 3,4 & 12,5 & 12,6 & 1,4 & 3,4 & 22,3 & 18,9 & 26,7 & 12,0 & 7,2 & 9,3 & 39,6 \\
\hline Não identificadas & 8,3 & 3,8 & 3,6 & 5,1 & 5,8 & 1,9 & 2,9 & 9,1 & 4,1 & 2,3 & 4,8 & 5,7 & 23,8 \\
\hline Média $\left(\right.$ sementes por $\mathrm{m}^{2}$ ) & 23.654 & 21.839 & 10.468 & 9.744 & 45.747 & 24.400 & 2.815 & 2.882 & 2.382 & 5.115 & 5.167 & 4.472 & 1.322 \\
\hline
\end{tabular}

${ }^{(1)}$ Em cada área, foram coletadas oito amostras compostas por quatro subamostras de 7,3 cm de diâmetro; L: lavoura contínua; LPL: lavoura-pastagem-lavoura; PLP: pastagem-lavoura-pastagem; P: pastagem contínua; C: preparo convencional do solo; D: semeadura direta; 1: adubação de manutenção; 2: adubação corretiva gradual. ${ }^{(2)}$ Espécies encontradas em maior densidade. ${ }^{(3)} P$. pedicellatum + P. polystachion + P. setosum. 
O nível de adubação afetou apenas a densidade de sementes nas áreas de cultivo LPLC e PLPC (Tabela 2). $\mathrm{Na}$ área de cultivo de LPLC, houve maior número de sementes com a adubação de manutenção do que com a adubação corretiva gradual. Isso ocorreu, possivelmente, em conseqüência do recobrimento mais rápido da área pela soja com a adubação corretiva gradual, o que dificultou o desenvolvimento das espécies de plantas daninhas. Na área de cultivo de PLPC, houve menor número de sementes com a adubação de manutenção do que com a adubação corretiva gradual.

As áreas com adubação corretiva gradual apresentaram maior número de espécies, excluindo-se as áreas de cultivo de PLP com semeadura direta, que apresentaram o mesmo número de espécies nos dois níveis de adubação (Tabela 2). Não são encontradas informações na literatura sobre o efeito da adubação, em relação ao número de espécies, em condições semelhantes às do presente estudo. Nicandra physaloides e Solanum americanum ocorreram em maior porcentagem de densidade de sementes em áreas com adubação corretiva gradual do que nas áreas de adubação de manutenção (Tabela 3). De acordo com Kissmann (2000), N. physaloides prefere solos com boa fertilidade, assim como $S$. americanum. Acanthospermum australe ocorreu em maior porcentagem da densidade de sementes em áreas com adubação de manutenção do que nas áreas de adubação corretiva gradual. Segundo Kissmann \& Groth (1999), A. australe se sobressai à maioria das espécies em solos com menor fertilidade.

\section{Conclusões}

1. Os sistemas de cultivo com presença de gramíneas forrageiras perenes apresentam menor densidade de sementes e número de espécies de plantas daninhas, no banco de sementes, em relação aos sistemas com culturas de lavoura.

2. A semeadura direta reduz a densidade de sementes no solo, em relação ao preparo convencional do solo, apenas em áreas intensamente perturbadas como as lavouras.

3. Na maioria das áreas, não há efeito do nível de adubação sobre a densidade de sementes no solo.

\section{Agradecimentos}

Ao pesquisador Antônio Carlos Gomes, pelo auxílio nas análises estatísticas; ao Institut de Recherche pour le Développement (IRD), Ministères des Affaires Etrangères (MAE), à Embrapa Cerrados, UnB e Capes, pelo apoio financeiro.

\section{Referências}

BELLINDER, R.R.; DILLARD, H.R.; SHAH, D.A. Weed seedbank community responses to crop rotation schemes. Crop Protection, v.23, p.95-101, 2004.

CHAUHAN, B.S.; GILL, G.; PRESTON, C. Influence of tillage systems on vertical distribution, seedling recruitment and persistence of rigid ryegrass (Lolium rigidum) seed bank. Weed Science, v.54, p.669-676, 2006.

COBUCCI, T.; PORTELA, C.M.O. Manejo de herbicidas no sistema Santa Fé e na braquiária como fonte de cobertura morta. In: KLUTHCOUSKI, J.; STONE, L.F.; AIDAR, H. Integração lavoura-pecuária. Santo Antônio de Goiás: Embrapa Arroz e Feijão, 2003. p.443-458.

EKELEME, F.; AKOBUNDU, I.O.; ISICHEI, A.O.; CHIKOYE, D. Cover crops reduce weed seedbanks in maize-cassava systems in Southwestern Nigeria. Weed Science, v.51, p.774-780, 2003.

FAVRETO, R.; MEDEIROS, R.B. Banco de sementes do solo em área agrícola sob diferentes sistemas de manejo estabelecida sobre campo natural. Revista Brasileira de Sementes, v.28, p.34-44, 2006.

GASPARINO, D.; MALAVASI, U.C.; MALAVASI, M.M.; SOUZA, I. Quantificação do banco de sementes sob diferentes usos do solo em área de domínio ciliar. Revista Árvore, v.30, p.1-9, 2006.

KISSMANN, K.G. Plantas infestantes e nocivas. 2.ed. São Paulo: BASF, 1997. v.1. 824p.

KISSMANN, K.G. Plantas infestantes e nocivas. 2.ed. São Paulo: BASF, 2000. v.3. 722p.

KISSMANN, K.G.; GROTH, D. Plantas infestantes e nocivas. 2.ed. São Paulo: BASF, 1999. v.2. 978p.

LÉGÈRE, A.; STEVENSON, F.C.; BENOIT, D.L. Diversity and assembly of weed communities: contrasting responses across cropping systems. Weed Research, v.45, p.303-315, 2005.

MENDONÇA, R.C.; FELFILI, J.M.; WALTER, B.M.T.; SILVA JÚNIOR, M.C.; REZENDE, A.V.; FILGUEIRAS, T.S.; NOGUEIRA, P.E. Flora vascular do Cerrado. In: SANO, S.M.; ALMEIDA, S.P. Cerrado: ambiente e flora. Planaltina: EmbrapaCPAC, 1998. p.289-556.

MOONEN, A.C.; BARBERI, P. Size and composition of the weed seedbank after 7 years of different cover-crop-maize management systems. Weed Research, v.44, p.163-177, 2004.

MURPHY, S.D.; CLEMENTS, D.R.; BELAOUSSOFF, S.; KEVAN, P.G.; SWANTON, C.J. Promotion of weed species diversity and reduction of weed seedbanks with conservation tillage and crop rotation. Weed Science, v.54, p.69-77, 2006.

PEREIRA, F.A.R.; VELINI, E.D. Sistemas de cultivo no Cerrado e dinâmica de populações de plantas daninhas. Planta Daninha, v.21, p.355-363, 2003. 
SEVERINO, F.J.; CARVALHO, S.J.P.; CHRISTOFFOLETI, P.J. Interferências mútuas entre a cultura do milho, espécies forrageiras e plantas daninhas em um sistema de consórcio. III - Implicações sobre as plantas daninhas. Planta Daninha, v.24, p.53-60, 2006.

SOSNOSKIE, L.M.; HERMS, C.P.; CARDINA, J. Weed seedbank community composition in a 35-year-old tillage and rotation experiment. Weed Science, v.54, p.263-273, 2006.
VILELA, L.; MACEDO, M.C.M.; MARTHA JÚNIOR, G.B.; KLUTHCOUSKI, J. Benefícios da integração lavoura-pecuária. In: KLUTHCOUSKI, J.; STONE, L.F.; AIDAR, H. Integração lavoura-pecuária. Santo Antônio de Goiás: Embrapa Arroz e Feijão, 2003. p.143-170.

ZIMDHAL, R.L. Weed ecology. In: ZIMDHAL, R.L. Fundamentals of weed science. San Diego: Academic Press, 1993. p.150-154.

Recebido em 25 de junho de 2007 e aprovado em 24 de setembro de 2007 